\title{
A Low-cost Media-Processor based Real-Time MPEG-4 Video Decoder
}

Jin-Hau Kuo, Ja-Ling Wu, Senior Member, IEEE, Jim Shiu*, and Kan-Li Huang

\author{
Communications and Multimedia Laboratory, \\ Department of Computer Science and Information Engineering, \\ National Taiwan University, Taipei, Taiwan, \\ *SetaBox Technology Corp. (STC), Taipei, Taiwan
}

\begin{abstract}
In this paper, by implementing a realistic MPEG-4 core profile decoder over the TriMedia ${ }^{T M}$ DSP chip [7], we investigate a serious of important issues related to the use of a media-processor to satisfy multimedia compression and processing requirement. The realistic MPEG-4 decoder optimized implementation by this DSP chip can support resolutions up to $4 C I F(720 \times 576)$ at $30 \mathrm{fps}$. The issues we investigated include the overview of characteristics of media-processor architecture [8-15], the speedup of the MPEG-4 decoder based on the TriMedia ${ }^{T M}$ DSP chip, multimedia co-processor or hardware accelerator based approaches, Very Long Instruction Word (VLIW) and Single Instruction Multiple data (SIMD) programming technique and the media-processor based solution for set-top box applications.
\end{abstract}

\section{INTRODUCTION}

Along with the high-speed development of semi-conductor technology, improvements in microprocessor architecture and performance have enabled general-purpose computer chips to process digital video, audio and graphics in recent years. But as the concept of Information Appliance (IA) has emerged, the similar functionality has shifted from the general-purpose to the so-called media-processor one. The processor is targeted to mass-produced consumer electronics devices, such as digital televisions, set-top boxes, DTV and as much qualifies as an embedded processor. Meanwhile, the progress of video compression techniques is maturing after years of efforts on development of many standard and de-facto codecs. The well-known moving picture expert group (MPEG) has worked out the newest version of video codec, MPEG-4 [1], possessing very low bit-rate and, yet acceptable quality. Recently, much research [2-6] has focused on considering the media-processor for these multimedia compression and processing requirements, such as the MPEG-4 decoder using a media-processor. The compelling reasons for it are flexibility and low-cost. A media-processor-based terminal architecture has to provide a cost effective (based on commodity pricing) execution environment for video applications because silicon resources are limited. Therefore, there are many challenges in designing and programming media-processors.

\section{THE HIERARCHY ACCELERATION AND THE RELATED METHODOLOGIES}

Two years prior to this work, we investigated several issues centered on the MPEG-1, 2 video decoder paired with the Intel MMX instruction set [16]. Relying on our previous findings, we first investigate the issue of speeding up the MPEG-4 decoder using the TriMedia ${ }^{T M}$ DSP chip. The steps of acceleration can be considered as a hierarchy stage shown in Fig. 1. From top to bottom are the stages of Algorithm, Instruction Set and Assembly Level, respectively. As expected, based on previous research, the inverse discrete cosine transform (IDCT) and motion compensation (MC) are the two most processing-intensive tasks of MPEG-4 video decoder (As shown in the performance profile in Fig. 2). The performance profile is obtained by porting a pure $\mathrm{C}$ code and non-accelerated MPEG-4 decoder over the TriMedia ${ }^{T M}$ DSP chip preliminary. After that, we demonstrated how to speed up MPEG-4 video decoder according to the VLIW architecture. First, we show how to use data and instruction parallelism by VLIW to enhance the inverse discrete cosine transform (IDCT), followed by then how to speed up the motion compensation (MC) module. Thirdly, we demonstrated how operations in the MPEG-4 video decoder are also enhanced by VLIW instruction set, as well as using the cache system of TriMedia ${ }^{T M}$ DSP chip to exploit data locality. The methods for speed-up IDCT can summarize as (1) Fast algorithm [17] (2) Loop unrolling to unroll IDCT source code, we can schedule this module into VLIW architecture more compactly (3) In-place matrix transpose - just transposing matrix in place to avoid the matrix transpose in IDCT module. (4) TriMedia ${ }^{T M}$ SIMD instruction. The approaches to optimize the MC module we used are as (1) Loop unrolling (2) TriMedia ${ }^{T M}$ SIMD instruction. By using SIMD instructions, we can do multiple motion compensation at a time. But some overheads caused by the lacks of some frequently used SIMD instruction supports by the Intel MMX \& SSE instruction set reduce the performance improvement (c.f. Fig. 3). These methods conform to the concept of the hierarchy stages of acceleration. 


\section{CONCLUSION}

We compared the similarities and dissimilarities of the Intel and TriMedia ${ }^{T M} D S P$ chip. The drawbacks and the further possible improvement of the DSP chip are also discussed. By cooperation with the SetaBox Technology Corp. (STC), we also investigated the media-processor in set-top box application. This includes the current and potential market, as well as making practical solutions for set-top box application.

\section{REFERENCES}

[1] ISO/IEC International Standard 14496, Information Technology - Generic Coding of Audio-Visual Objects Part 1: System, Part 2: Visual, Part 3: Audio, Part 5: Reference software, Part 6: DMIF. 2000.

[2] Le, T.M., Nita, A., Giernalczyk, E., Denny Wong, Tuan Ho, "A low-power and scalable MPEG-4 solution for wireless communications," in IEEE Int. Conf. Consumer Electronics, 2001

[3] Fermo, A., Sicuranza, G.L., Pahor, V., "Hardware-oriented region based algorithm for low power motion estimation," in IEEE Proceedings of the $2^{\text {nd }}$ International Symposium on Image and Signal Processing and Analysis, 2001

[4] Ramkishor, K., Gunashree, V., "Real time implementation of MPEG-4 video decoder on ARM7TDMI," in IEEE Proceedings of International Symposium on Intelligent Multimedia, Video and Speech Processing, 2001

[5] Burleson, W., Jain, P., Venkatraman, S., "Dynamically parameterized architectures for power-aware video coding: motion estimation and DCT," in IEEE Proceedings of the $2^{\text {nd }}$ International Workshop on Digital and Computational Video, 2001

[6] "Special Issue on Multimedia Implementation," Call For Papers in IEEE Transaction on Circuits and System for Video Technology, to appear on March, 2002

[7] Philips TriMedia ${ }^{\mathrm{TM}}$ Software Development Environment Version 2.1.

[8] Pol, E.J.D., Aarts, B.J.M., van Eijndhoven, J.T.J., Struik, P., Sijstermans, F.W., Tromp, M.J.A., van de Waerdt, J.W., van der Wolf, P., "TriMedia CPU64 application development environment," in IEEE Int. Conf. On Computer Design,
1999

[9] Van Eijndhoven, J.T.J., Sijstermans, F.W., Vissers, K.A., Pol, E.J.D., Tromp, M.I.A., Struik, P.; Bloks, R.H.J., van der Wolf, P., Pimentel, A.D., Vranken, H.P.E., "TriMedia CPU64 architecture," in IEEE Int. Conf. On Computer Design, 1999

[10] Equator Technologies Inc. http://www.equator.com

[11] Texas Instrument Technologies Inc. http://www.ti.com

[12] Intel Architecture Optimization - Reference Manual

[13] Intel Architecture MMX New Instruction Technology

[14] Intel Architecture SSE (Katmai) New Instruction Technology

[15] MPEG Macroblock Parsing and Pel Reconstruction on an FPGA-augmented TriMedia Processor

[16] Yi-Shin Tung, Chia-Chiang Ho, Ja-Ling Wu, "The MMX-based IDCT and MC Algorithms for Real-Time Pure Software MPEG Decoding," ICMCS'99, Florence Italy

[17] Y. Arai, T.Agui, and M.Nakajima, "A Fast DCT-SQ Scheme for Images", Trans. Of the IEICE, E71(11):1095, Nov 1988.

\begin{tabular}{|c|c|c|c|c|c|c|}
\hline function & Executions & Total Cyel & os (z) & I\$ Cyeles & of cyclos & \\
\hline _Copy日lockHorVe & 614979 & 437293858 & 10.04 & 13210996 & 2158627 & \\
\hline _transferJOCT_add_generic & 530893 & 371578962 & 8.53 & 59893761 & & \\
\hline _CopyMBlockHorVer_gener ie & 138193 & 358546332 & 8.23 & 5640602 & 49494804 & * \\
\hline _idctcos & 4643804 & 331471600 & 3.61 & 32027377 & 4685131 & \\
\hline _CopyllBlock_generic & $1872 \theta 7$ & 268481623 & 6.17 & $1781040 \theta$ & 58786814 & * \\
\hline & 459416 & 188806149 & 4.34 & 4119525 & 66620194 & 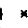 \\
\hline _nencpy & 403967 & 183496477 & 4.27 & 185861 & 126724480 & \\
\hline macroblock_p_uop & 499515 & 181565938 & 4.17 & 57867577 & 29312981 & \\
\hline blockInter & 538081 & 159139587 & 3.65 & 53325557 & $237924 \theta$ & \\
\hline gotrudeata & 1213315 & 143874323 & 3.29 & 80980299 & 5427393 & \\
\hline _idet_gener & 586477 & 106495177 & 2.45 & 11282312 & & \# \\
\hline CopyBjockHor_goner ic & 153838 & 99693578 & 2.29 & 18988446 & 19714491 & * \\
\hline _CopyllslockHor_generic & 44749 & 99410594 & 2.28 & 3198491 & 15221682 & $2 \times$ \\
\hline _uld_inter_det & 1219352 & 97909204 & 2.25 & 37190212 & 1991 & \# \\
\hline idetrow & 4643819 & 96552869 & 2.22 & 13489885 & & : \\
\hline find_plu & 1213398 & 95485144 & 2.19 & 27141888 & 19140813 & \\
\hline 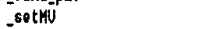 & 666648 & 94849024 & 2.18 & 43882182 & 7298152 & \\
\hline nextbits_bytealigned & 1001767 & 94245995 & 2.16 & 29383969 & $\theta$ & \\
\hline & 492475 & 90302893 & 2.07 & 43219392 & 14766861 & \\
\hline recon_t & 1752651 & 78946290 & 1.81 & 4831733 & 28541300 & \\
\hline CopyBlockUer_generic & 123704 & 76469683 & 1.76 & 2116745 & 18810997 & 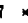 \\
\hline
\end{tabular}

Figure 2: The preliminary performance profile of the non-accelerated MPEG-4 decoder over the TriMedia ${ }^{T M}$ DSP chip: The columns marked by the symbol * and \# are the DSP cycles: instruction cycles and data cycles, executed by the MC and IDCT, respectively.

Algorithm level

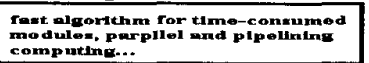
Instruction set level Heing the complifer tools supported algortiche bered on tho dodlcated intruction eet supported by the DSF $\downarrow$ Assembly level makcing bp for tho drawbacker the Umbe-conemed module. In

Figure 1: The hierarchy stages of acceleration and the related methodologies

\begin{tabular}{|c|c|c|c|}
\hline \multicolumn{4}{|c|}{ Data Type : 8 bits signed or uneigned intger Operation } \\
\hline Operation (customer ops) & Yes & No & Purpose \\
\hline QUADAVG & $T$ & & Unsigned byte-wise quad average \\
\hline FUNSHIFTI & $t$ & & Furnel-shift l-hyte \\
\hline FUNSHEFT2 & 1 & & Furnel-shift 2-byte \\
\hline FUNSHIFT3 & 1 & & Funnel-shift 3-byte \\
\hline DSPUQUADADDUI & t & & Quad clipped add of uns ignedsigned bytes \\
\hline DUALICLIPI & 1 & & Dual-16 dip signed to signed \\
\hline QUADD & & 1 & 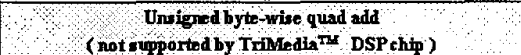 \\
\hline QUADAVGN & 4 & 1 & 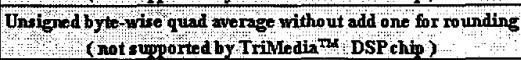 \\
\hline
\end{tabular}

Figure 3: The Used Customer Operations in MC 